\title{
Evolution of the distribution of dispersal distance under distance-dependent cost of dispersal
}

\author{
F. ROUSSET* \& S. GANDON $\dagger$ \\ *Institut des Sciences de l'Évolution, Place E. Bataillon, Montpellier Cedex, France \\ †Centre d'Etude sur le Polymorphisme des Micro-Organismes, IRD, Montpellier Cedex 1, France and, Institute for Cell, Animal and Population Biology, \\ University of Edinburgh, Ashworth Laboratories, Edinburgh, UK
}

\section{Keywords:}

dispersal;

evolutionarily stable strategy;

isolation by distance;

kin selection.

\begin{abstract}
We analyse the evolution of the distribution of dispersal distances in a stable and homogeneous environment in one- and two-dimensional habitats. In this model, dispersal evolves to avoid the competition between relatives although some cost might be associated with this behaviour. The evolutionarily stable dispersal distribution is characterized by an equilibration of the fitness gains among all the different dispersal distances. This cost-benefit argument has heuristic value and facilitates the comprehension of results obtained numerically. In particular, it explains why some minimal or maximal probability of dispersal may evolve at intermediate distances when the cost of dispersal function is an increasing function of distance. We also show that kin selection may favour long range dispersal even if the survival cost of dispersal is very high, provided the survival probability does not vanish at long distances.
\end{abstract}

\section{Introduction}

Dispersal affects both the population dynamics and the population genetics of species. Reciprocally, the dynamics and the genetics of species are likely to act on this behaviour. The forces that may select for higher probabilities of dispersal include (1) temporal variability in the habitat (Van Valen, 1971); (2) avoidance of inbreeding depression (Bengtsson, 1978); (3) kin competition (Hamilton, 1964; Hamilton \& May, 1977). As a first approximation, the evolution of dispersal may be described by a balance between these forces and a cost due to either increased mortality during the dispersal phase or during the settling period in a novel habitat. All these factors are often pooled in a single parameter: the cost of dispersal. Further, it is very convenient to assume an island model of migration, where dispersers are redistributed randomly among the different populations. Under this simplifying assumption dispersal is fully characterized by a single parameter, the dispersal probability, which measures the fraction of the progeny leaving its natal site.

Correspondence: Institut des Sciences de l'Évolution, CC065, USTL, Place E. Bataillon, 34095 Montpellier Cedex 05, France.

Tel.: +33 467 144630; fax: +33 467 143622;

e-mail: rousset@isem.Univ-montp2.fr
However, dispersal does not usually follow an island model and the distribution of dispersal distances is often of interest in itself. It is known that the shape of the tail of the distribution of dispersal distance (long-distance dispersal) determines the rate of spread of colonizing populations as shown by studies of disease epidemics or of post-glacial rates of advance in many plant species (e.g. (Mollison, 1991; Kot et al., 1996; Shigesada \& Kawasaki, 1997; Clark et al., 2001). Although the evolution of the dispersal probability has been analysed assuming dispersal is localized, as in 'stepping stone' models, it was still assumed that the distribution of dispersal distance itself was fixed and that the cost was independent of distance (Comins, 1982; Gandon \& Rousset, 1999). However, the distribution of dispersal distances itself is subject to selection. Here, our analysis assumes that there is no $a$ priori constraint on the dispersal distribution: any distribution is a possible strategy. This contrasts to models where a more restricted family of dispersal distributions is considered (Ezoe, 1998; Bolker \& Pacala, 1999; Gandon \& Rousset, 1999; Harada, 1999).

The different selective pressures acting on the shape of the dispersal distribution vary with the dispersal distance (Ronce et al., 2001). In this paper we provide a formal basis for the evolution of dispersal distribution in the case 
where only two forces are acting: kin competition and distance-dependent cost of dispersal. In a stable and homogeneous habitat there is always a benefit to disperse far because it decreases the risk of competing with related individuals. On the other hand, the cost of dispersal may be an increasing function of dispersal distance. This cost will have a direct effect on the evolution of dispersal but it will also feed back on the distribution of genetic variation and, therefore on kin competition and the benefit of long-distance dispersal. Kin competition and distance-dependent cost of dispersal are ubiquitous forces acting on this distribution and our aim is to provide a model which may serve as a reference against which one can evaluate the importance of additional factors that may affect the evolution of dispersal distributions.

We first present a general treatment of the evolution of dispersal with any number of demes and for any function of dispersal cost. This analysis is then used in one- (1D) and two-dimensional (2D) models to study the effects of two factors on the evolution of dispersal: (1) deme size; (2) the shape of the cost of dispersal function. We find that long-distance dispersal is selected for even if the survival cost of dispersal is very high, provided the probability of survival does not vanish at long distances. This analysis relates the evolutionarily stable dispersal distribution to a given distribution of distance-dependent cost of dispersal.

\section{The model}

\section{Formulation}

The phenotype of an adult is described by the dispersal probabilities $\left(\ldots, d_{\mathbf{i}}, \ldots\right) \equiv \mathbf{d}$ of its juveniles to all possible distances $\mathbf{i} \equiv(x, y)$, as shown, e.g. in Fig. 1 .

\section{Life cycle}

The habitat consists of a finite number, $n$, of demes on a lattice organized in a 1D or 2D torus of sizes $n_{x}$ and $n_{y}$ in each dimension $\left(n=n_{x} n_{y}\right)$. On the lattice, individuals are separated by distances $\mathbf{i}=(x, y)$ for discrete values of $x$ and $y$. All demes are occupied with an equal number, $N$, of haploid individuals. We assume the following lifecycle: (i) reproduction occurs and a large number of juveniles are produced. (ii) Mutation occurs at a rate $u$. (iii) Juvenile dispersal occurs under a given distribution of dispersal distances. (iv) Dispersing offspring incur the cost of dispersal, $c_{\mathbf{i}}$, which is a function of dispersal distance i. (v) Adults die. (vi) Offspring compete for the $N$ available sites in each deme.

\section{Isolation by distance}

Some genetic differentiation results from localized dispersal. Differentiation may be measured by the probability of identity by descent in a mutation-drift model under the

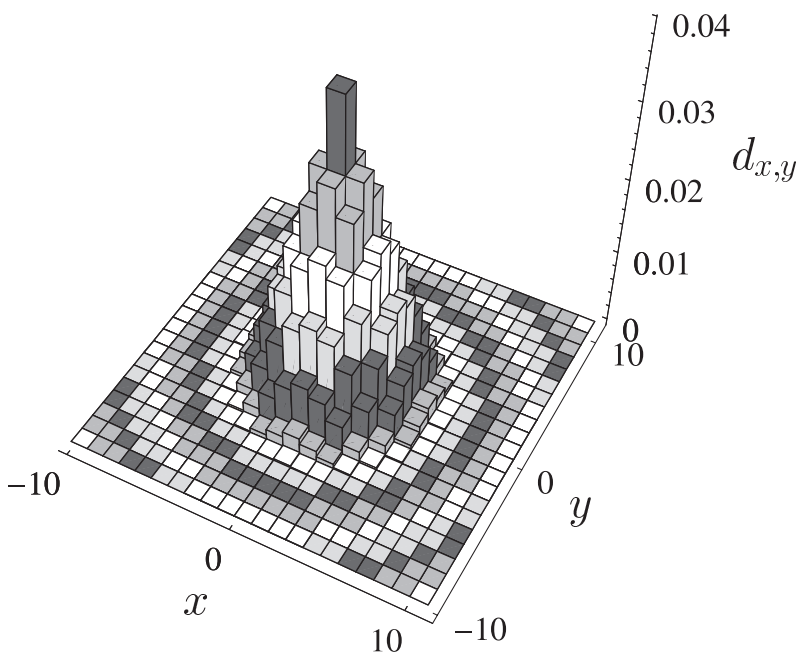

Fig. 1 A two-dimensional dispersal distribution. In comparison with this Figure, Fig. 2 presents distributions binned in intervals $[0,0.5],[0.5,1.5], \ldots,[9.5,10.5]$, obtained by summing all probabilities of dispersal at Euclidian distances within ranges

$[0,0.5],[0.5,1.5], \ldots,[9.5,10.5]$, shown here in different shadings. This example shows the dispersal distribution for a linear cost of dispersal (Fig. 2c), with $N=1$.

given life-cycle. Under the assumptions of the model, such probabilities depend on the distance $\mathbf{i}$ (relative position) between the genes, but not on their absolute position in space. We note $Q_{\mathbf{i}}$ the probability of identity of genes from two juveniles sampled in demes separated by distance $\mathbf{i}$, after dispersal and before competition.

\section{Conditions for convergence stability}

Here we define conditions for convergence towards an evolutionarily stable dispersal distribution. We consider selection among 'symmetrical' strategies, i.e. given a dispersal probability at some distance $(x, y)$, we assume the same dispersal probabilities at distances $(x,-y)$, $(-x, y)$, and $(-x,-y)$ (as show, e.g. in Fig. 1). We write $d_{ \pm x, \pm y}$ for the sum of dispersal probabilities at these different symmetrical distances. Each phenotype may thus be described by $\left(d_{0,0}, d_{ \pm 1,0}, d_{0, \pm 1}, d_{ \pm 1, \pm 1}, \ldots\right)$, where $d_{0,0}$ is the fraction of philopatric offspring. No constraint is imposed on these probabilities, except that they sum to 1 .

Consider first two alleles $a$ and $A$, strategies $\mathbf{d}^{a}$ and $\mathbf{d}^{A}$ differ as follows. For allele $A$ the dispersal probability $d_{D}^{A}$ at some distance $D \equiv\left(D_{x}, D_{y}\right)$ is higher than the dispersal probability $d_{D}^{a}$ at this distance for allele $a$. For allele $A$, dispersal probabilities to all other distances are reduced in proportion to $\left(1-d_{ \pm D_{x}, \pm D_{y}}^{A}\right) /\left(1-d_{ \pm D_{x}, \pm D_{y}}^{a}\right)$. Here $d_{D}$ may be noted simply $z$, with values $z^{a}$ or $z^{A}$ for individuals bearing allele $a$ or $A$. The strategy of a focal individual will be noted $z_{\bullet}$, and the average strategy of individuals at distance $\mathbf{i}$ relative to the focal individual will be written $z_{\mathbf{i}}$. 
The expected number of adult offspring of a focal individual can then be described by a fitness function $w\left(z_{\bullet}, \mathbf{z}, D\right)$, function of the focal individual's strategy and of the strategies of all its competitors $\mathbf{z} \equiv\left(\ldots, z_{\mathbf{i}}, \ldots\right)$ over all distances from the focal individual on the lattice. Following Gandon \& Rousset (1999), $w$ can be expressed in terms of the relative number $g_{\mathbf{i} \rightarrow \mathbf{j}}$ of juveniles from deme $\mathbf{i}$ (relative to the focal deme) in competition for deme $\mathbf{j}$ (relative to the focal deme) after dispersal:

$$
w\left(z_{\bullet}, \mathbf{z}, D\right) \equiv \sum_{\mathbf{j}} \frac{g_{\mathbf{0} \rightarrow \mathbf{j}}\left(z_{\bullet}, D\right)}{\sum_{\mathbf{i}} g_{\mathbf{i} \rightarrow \mathbf{j}}\left(z_{\mathbf{i}}, D\right)}
$$

Each term of the sum over $\mathbf{j}$ represents the expected number of offspring of the focal in the deme at distance $\mathbf{j}$ from the focal parent. Each of these terms is the ratio of the focal individual's juveniles (the numerator of the ratio) relative to all juveniles that come in competition for this deme (the denominator of the ratio). The functions $g_{\mathbf{i} \rightarrow \mathbf{j}}$ are detailed in eq. 5 in the Appendix. Selection on the $A$ allele can then be measured by

$$
\phi_{D}\left(z^{a}\right) \equiv \lim _{u \rightarrow 0} \frac{S_{D}\left(z^{a}\right)}{1-Q_{\mathbf{0}}}
$$

where

$$
S_{D}\left(z^{a}\right) \equiv \sum_{\mathbf{i}}\left(Q_{\mathbf{i}}^{\prime}-1\right) \partial_{z_{\mathbf{i}}} w\left(z_{\bullet}, \mathbf{z}, D\right)
$$

(Rousset \& Billiard, 2000). In this expression, effects of neighbours with average phenotype $z_{\mathbf{i}}$ on the fitness of an $A$-bearing individual are measured by the derivative with respect to $z_{\mathbf{i}}$, evaluated in $z^{a}$ for all $z$ variables. Each such effect is weighted by the probability $Q_{i}^{\prime}$, which measures the covariation between the phenotype of the focal individual and the phenotypes represented by each variable $z_{\mathbf{i}}$. Hence $Q_{\mathbf{i}}^{\prime}$ is the identity between the focal adult and a random adult at distance i relative to the focal one.

In particular, $z_{\mathbf{0}}$ represents the average phenotype in the focal deme, including the focal individual. Hence $Q_{\mathbf{0}}^{\prime}$ is the identity between the focal adult and a random adult in the focal deme. With probability $1 / N$, this random adult is the focal individual itself. The identity between different individuals when adults (i.e. after competition in the life cycle) is identical to their identity when sampled younger (right before competition), which was noted $Q_{\mathbf{i}}$. Hence $Q_{\mathbf{0}}^{\prime}=1 / N+(1-1 / N) Q_{\mathbf{0}}$ and $Q_{\mathbf{i}}^{\prime}=Q_{\mathbf{i}}$ for $\mathbf{i} \neq \mathbf{0}$.

A dispersal probability $z^{a}$ is stable against a mutant $A$, with the effects described above on the dispersal distribution, if it obeys either of the following conditions: (i) $z^{a}=0$ and $\phi_{D}(0) \leq 0$ (dispersal is counterselected at distance $D$ ), or (ii) $z^{a}>0$ and $\phi_{D}\left(z^{a}\right)=0$ (some intermediate dispersal probability is selected). Now consider the stability of a strategy against mutants that may alter the dispersal distribution in a more complicated way, e.g. by increasing dispersal at several distances. For all dispersal distances $D, D^{\prime}$ at which it has nonzero levels of dispersal, the strategy is stable only if $\phi_{D}=\phi_{D^{\prime}}=0$ (see Appendix). Using expressions for $\phi_{D}$ derived in the Supplementary Appendix (see section Supplementary material), this implies

$$
\left(1-c_{D}\right)\left(1-\sum_{\mathbf{i}} m_{D+\mathbf{i}} Q_{\mathbf{i}}^{\prime}\right)=\left(1-c_{D^{\prime}}\right)\left(1-\sum_{\mathbf{i}} m_{D^{\prime}+\mathbf{i}} Q_{\mathbf{i}}^{\prime}\right)
$$

where the $m^{\prime}$ s are the backward dispersal probabilities $m_{\mathbf{j}} \equiv g_{\mathbf{0} \rightarrow \mathbf{j}} / \sum_{\mathbf{i}} g_{\mathbf{i} \rightarrow \mathbf{j}}$, i.e. the probabilities that an adult was born $\mathbf{i}$ demes away, by contrast with the 'forward' rates $d_{\mathbf{i}}$ which describe where juveniles go.

\section{A cost-benefit argument}

Equation 4 leads to an intuitive cost-benefit argument. Dispersal at a distance $D$ is associated with two types of costs: (i) $c_{D}$ is the direct cost (cost paid by the disperser) whereas (ii) $\sum_{\mathbf{i}} m_{D+\mathbf{i}} Q_{\mathbf{i}}^{\prime}$ measures the indirect cost because of the competition (in deme $D$ ) with related individuals (from demes i steps apart). The above relation shows that, at the ESS, the product of direct and indirect benefits ( 1 -costs) should be the same at different dispersal distances. If the overall benefits associated with a particular dispersal distance were higher, a mutant strategy with higher dispersal at such distance could invade. The convergence stable distribution of dispersal cannot be replaced by any mutant and is characterized by an equilibration of the fitness gains among all the different dispersal distances.

The above cost-benefit argument yields some predictions regarding the shape of the convergence stable dispersal distribution. Let us focus on an organism sending all the dispersers at the same distance. All the dispersed offspring produced in a given deme (i.e. relatives) will compete against each other after the dispersal phase, leading to a large indirect cost of dispersal. The indirect cost of dispersal would be lower if these offspring dispersed at different dispersal distances. In other words, there is an inclusive benefit to spread the dispersers in different demes. Hence, if the cost of dispersal does not increase with distance, dispersal should follow an island mode of dispersal, where individuals that leave their natal deme are distributed randomly among all other demes.

However, the direct cost of dispersal is likely to be an increasing function of distance and, consequently, will select for less dispersal at higher distances. On the contrary the indirect cost will decrease with distance if there is lower dispersal at higher distances, because probabilities of identity will decrease with distance (genetic isolation by distance). This will select for more dispersal at higher distances. The magnitude of these different effects will be investigated below. 


\section{Finding the ES dispersal distribution}

The analysis of the evolution of the distribution of dispersal distances can be viewed as the analysis of the coadaptation of dispersal probabilities at different distances. The above cost-benefit analysis has heuristic value but it does not directly yield a quantitative prediction because the probabilities of identity are themselves a function of the dispersal distribution. In the Appendix we show how to construct an iterative algorithm to find the ES distribution of dispersal distances.

We note that this algorithm could be used for other purposes than in this paper. In particular we may constrain the evolution of dispersal to a given range of dispersal distances. Iterating the algorithm from an initial distribution with nonzero dispersal within this range and zero dispersal outside leads to the optimal distribution under such constraints.

We will use this algorithm to analyse the effects of (i) the size of the demes (ii) the shape of the cost function and (iii) the shape of the habitat (one or two dimensions). We explored the effect of the cost of dispersal through five different shapes of dispersal function: (1) an 'island' cost of dispersal where the cost is independent of dispersal distance as in the classical island model; (2) a 'saturating' cost as function of distance; (3) a 'linear' increase of the cost with distance; (4) an 'accelerating' cost; (5) a 'stepped' function where the cost increases step by step. Figure 2 presents some results: the convergence stable dispersal distribution, the average dispersal distance, the mean squared distance and kurtosis of the distribution. Dispersal can be described as the probability distribution of dispersal at some vectorial distance $(x, y)$, but it is also often described as the distribution of Euclidian distance $\sqrt{x^{2}+y^{2}}$. Further, dispersal data are often binned in distance classes. Hence we will show binned distributions, computed by summing such probabilities into bins corresponding to different ranges of Euclidian distance (see Fig. 1).

Result 1 (Deme size): Not surprisingly, larger deme size decreases the strength of kin competition at a natal site, which reduces both the dispersal probability (see also Taylor, 1988; Gandon \& Rousset, 1999) and (with the exception of the island cost of dispersal) the average dispersal distance (Fig. 2).

Result 2 ('Island' cost of dispersal): As expected from the qualitative argument presented in the previous subsection, we find that an 'island' cost of dispersal (Fig. 2a) selects for an island mode of dispersal. A formal proof of this result can be obtained (see Supplementary Appendix).

Result 3 (intermediate minimum cost): Another easily understood result is that when the cost of dispersal is minimized at some dispersal distance, the distribution of dispersal is also maximized at an intermediate distance (not shown). The maximum dispersal probability may be slightly further than the distance of this minimal cost, as a result of kin competition effects.
Result 4 (slow increase of cost): Less trivially, we find that if the direct cost increases more slowly than the indirect cost decreases with distance, it is possible to have a local maximum of dispersal at an intermediate distance. For example, when the cost of dispersal increases step by step (Fig. 2e) the distribution of dispersal evolves towards a saw-like shape where peaks of dispersal occur at the end of each step. On each step the direct cost does not vary whereas the indirect cost decreases with distance (because of the decrease of genetic identity with distance). This explains the evolution of higher dispersal at the end of the steps.

The example of the stepped cost function shows that if the cost sharply increases at some threshold distance, the optimal strategy allocates more dispersal right before this threshold. It should be noted that there is a simple mechanism which can generate a distribution with a maximum followed by a sharp decline at larger distances: ballistic dispersal (Stamp \& Lucas, 1983; Neubert et al., 1995). Ballistic dispersal may be understood as a mechanism with a given cost independent of realized dispersal distance up to a maximum distance imposed by this mechanism, and with a maximal dispersal probability at this maximum distance. Thus, it may be understood as a way of minimizing kin competition under constraints close to those described by a stepped cost function. Airborne dispersal can also generate distributions with a maximum at an intermediate distance, e.g. the lognormal distribution (Stoyan \& Wagner, 2001, and references therein).

Another example is illustrated in the one-dimensional case when the cost saturates (Fig. 2b). In this case, the direct effect of the cost increases more slowly than the indirect cost of competing with relatives decreases. This yields a local minimal probability of dispersal at an intermediate distance.

When the cost of dispersal increases monotonically with distance (Fig. 2b-d) the evolutionary outcome depends on the increase of the cost function. As expected, a 'saturating' function of the cost of dispersal (Fig. 2b) yields much more long-distance dispersal than an 'accelerating' function (Fig. 2d). It is shown in the Supplementary Appendix (eq. A.29) that in one dimension, if $1 /\left(1-c_{\mathbf{i}}\right)$ increases faster than distance $\mathbf{i}$, dispersal must be zero beyond some distance, so that there is no long-distance dispersal. This is because the relative quantitative effects of the cost of dispersal and of the benefits of avoiding competition between relatives are given by a comparison of $1 /\left(1-c_{\mathbf{i}}\right)$ to a measure of relatedness that varies linearly with distance (Rousset, 1997). In two dimensions we have a similar result except that the same measure of relatedness increases as the logarithm of distance. In other words, as relatedness decreases more slowly with distance in two dimensions, it pays less to disperse further away. Thus, the condition for the existence of long distance dispersal is more restrictive in two than in one dimension. 
$1 \mathrm{D}$

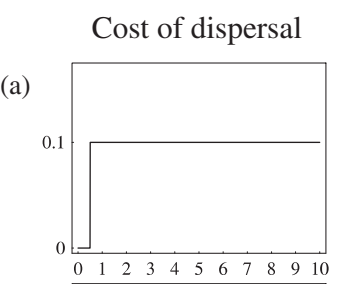

(b)

(c)
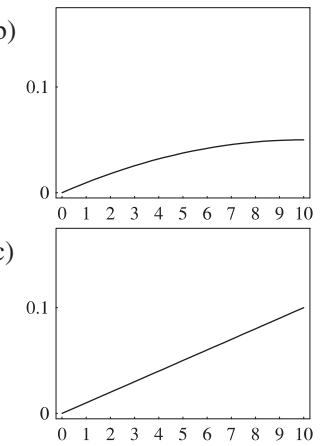

(d)

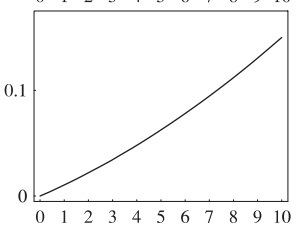

(e)

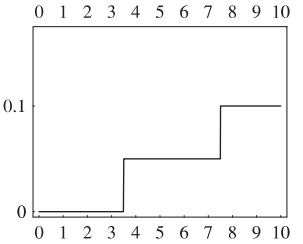

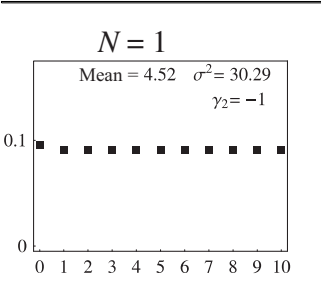
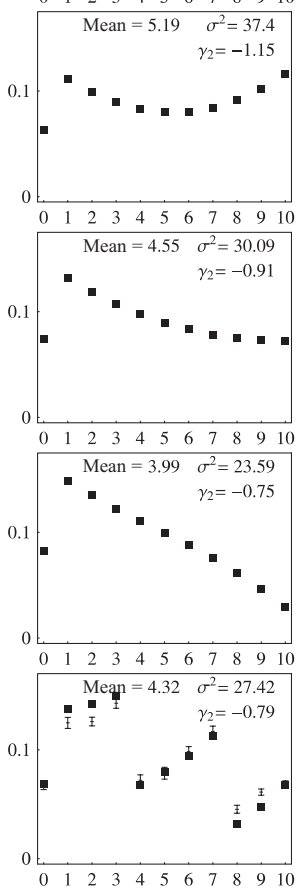

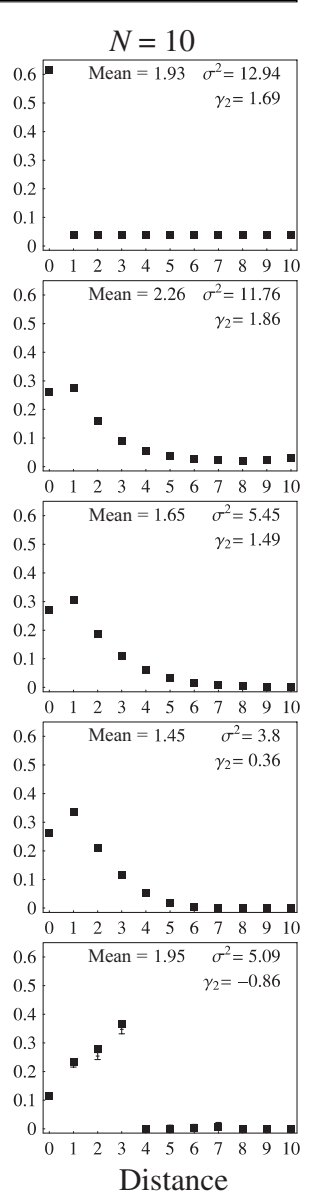

2D
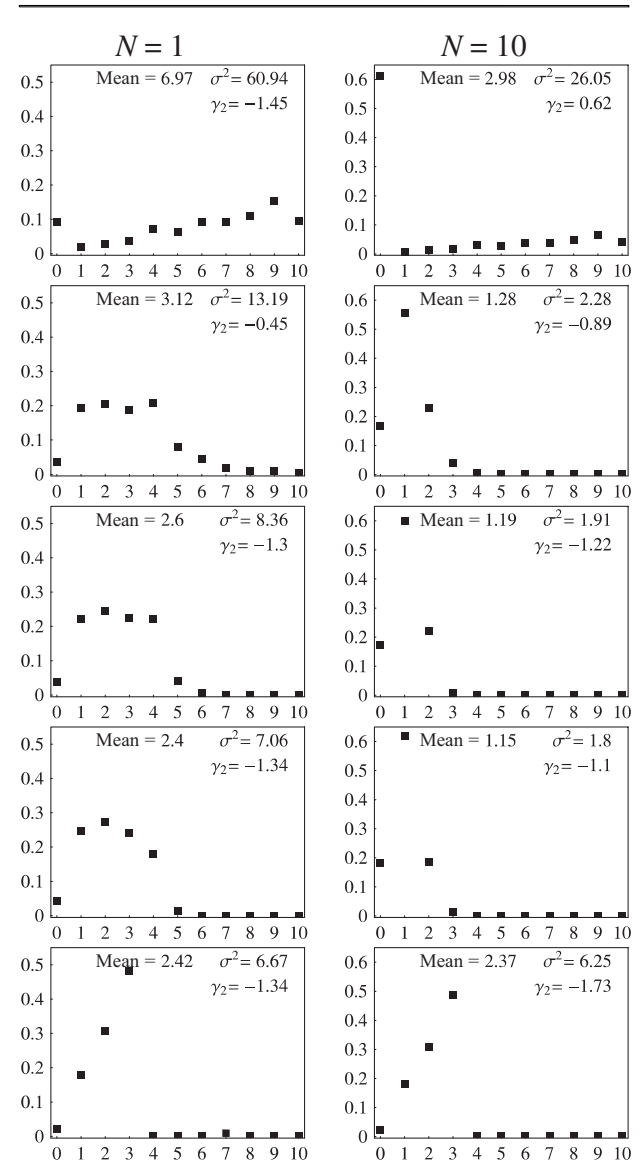

Fig. 2 Evolutionarily stable distributions of dispersal distance. Dispersal distributions are shown for different costs of dispersal functions (first column) and different deme sizes $(N=1$ or 10$)$ in one and two dimensional habitats ( 150 and $20 \times 20$ demes, respectively). In $1 \mathrm{D}$ case we obtained a maximal dispersal distance equal to 10 to facilitate comparison with the $2 \mathrm{D}$ case, by setting the cost to at least 0.5 at larger distances. In the two dimensional case we show the binned distribution, as explained in Fig. 1. Numerical computations were done using Mathematica (Wolfram, 1999). For each distribution, the mean dispersal distance ('mean'), mean squared dispersal distance $\left(\sigma^{2}\right)$ and kurtosis $\left(\gamma_{2}\right)$ are also given. For one of the costs of dispersal function (last row) we plot the results of an individual-based simulation in a one dimensional habitat with $150(N=10)$ or $500(N=1)$ demes [mean \pm standard error, computed by the batching method for Markov chains, Hastings (1970); for 20 batches of $10^{6}$ generations].

Result 5 (Evolution of long distance dispersal): If the survival probability does not vanish at long distances, then long distance dispersal is selected for. This is a consequence of the previous result 4 , as in this case $1 /\left(1-c_{\mathbf{i}}\right)$ increases too slowly at long distances to select against dispersal.

Individual based simulations have also been done in some cases as an independent way of obtaining the ES dispersal distribution. These simulations confirmed the results of numerical computation (Fig. 2e) and showed that evolution yields only one optimal distribution of dispersal (e.g. no stable polymorphism). Some differences between numerical and simulation results can result from the recurrent introduction of new genotypes through mutation (in the simulation we assumed a mutation rate equal to $\left.2.5 \times 10^{-4}\right)$. Indeed, higher mutation rates tend to bias the distribution of dispersal distance towards a distribution with identical dispersal probabilities at each distance. Nevertheless, these differences were small.

\section{Discussion}

\section{What can be inferred from data?}

Beyond quantifying the general trends expected from the qualitative arguments noted in the previous Section, our numerical analysis suggests some additional results. The 
above examples show that different functions of the cost of dispersal yield very different distributions of dispersal. Available data on the distribution of dispersal also show a high diversity. Even if dispersal distributions are often leptokurtic, i.e. have positive kurtosis (e.g. reviews and data in Endler, 1977; Portnoy \& Willson, 1993; Clark et al., 1999), many seed dispersal distributions have been found to have low kurtosis (Clark et al., 1999). The diversity of dispersal behaviours may thus be explained by the diversity of distance-dependent cost of dispersal.

However, the kurtosis of observed dispersal distributions is often much higher than the one found in the above numerical examples. This suggests that the actual cost of dispersal generally increases much more slowly than assumed in computing these examples. Little is known about the empirical relation between the cost of dispersal and distance. Qualitatively different relationships have been found. In some cases, the fitness costs induced by parasites have been shown to decrease with the distance of origin of hosts (Kaltz \& Shykoff, 1998). Some studies of 'inbreeding' and 'outbreeding depression' have found minimal costs at an intermediate distance (review in Waser, 1993; Ronsheim, 1997). Distance and/or density-dependent predation or parasitism (e.g. Janzen, 1970; Packer \& Clay, 2000) may also result in maximal costs at short distances. Our analysis can be used to infer the cost of dispersal function from observed distributions of dispersal distance (see Section 3 of the Supplementary Appendix). It would thus be particularly interesting to measure the cost of dispersal function and to compare it with the one inferred from the dispersal distribution. Any discrepancy between these two functions would demonstrate that the simplifying assumptions of our model are not met and that other forces are acting on the evolution of dispersal distance.

\section{Complications}

The above analysis shows that different costs of dispersal functions will act both directly (survival of dispersers) and indirectly (through its effect on relatedness). This second, indirect effect explains the occurrence of some minimal probability of dispersal at intermediate distances. However, in plants, relatedness is likely to depend not only on seed dispersal (probabilities and costs of dispersal) but also on pollen dispersal. Genetic isolation by distance will be reduced by pollen dispersal, and our results could be viewed as an overestimation of seed dispersal probabilities when pollen dispersal occurs. The distribution of pollen dispersal distance is also likely to evolve, and it would be interesting to further investigate the coevolution between seed and pollen dispersal. Another complicating factor is dormancy, which also reduces competition between relatives, along with other consequences, and may be negatively correlated with dispersal abilities (e.g. Olivieri \& Berger, 1985; Venable \& Brown, 1988; Willson, 1993).
The heterogeneity of the habitat may also lead to more complicated behaviours. Using an individual-based model, Savill \& Hogeweg (1998) showed that the evolution of dispersal distance may lead to a coexistence of short and long distance dispersal strategies. This polymorphism is because of the fact that they assumed that dispersal occurred in a two-dimensional lattice with a boundary. Individuals which cross the boundary are lost from the system. Therefore, near the boundary, the shorter the dispersal distance, the fewer offspring will be lost. In the centre of the lattice long distance dispersal is selected to avoid kin competition. Therefore, the result is a consequence of the cost of dispersal being variable in space.

Habitat heterogeneity may also arise from other processes. First, the uneven spatial distribution of individuals (resulting, e.g. from various extinction regimes) may generate different types of landscapes. If individuals are clustered, long-distance dispersers will have a higher chance (relative to short-distance dispersers) to settle in empty sites. This may select for longer dispersal distance than in homogeneous landscapes. Secondly, spatial heterogeneity may also emerge from intrinsic heterogeneity in the quality of the habitat. If there is spatial autocorrelation in this quality, long distance dispersers will, on average, reach unsuitable habitats, introducing a cost to long-distance dispersal from any habitat. Such extra costs of dispersal are likely to select for lower average dispersal distance (Hovestadt et al., 2001, Fig. 4). On the other hand, this cost of dispersal reaches a maximum below $l$ at moderately large distances. From our results we then expect that there will be long distance dispersal. The same result was observed in Hovestadt et al.'s simulations. The occurrence of local adaptation will have similar consequences. In the latter case, dispersal (the dispersal probability and the distribution of dispersal distances) may feed-back on the degree of local adaptation. Dispersal could either prevent (Slatkin, 1987) or enhance (Gandon et al., 1996) local adaptation depending on the temporal variability of the environments and other factors.

\section{Concluding remarks}

We have defined a set of tools to find the optimal dispersal distribution as a function of the cost of dispersal distance. With this approach, any cost function and any dispersal distribution may be considered. This general model has been analysed in order to explore the effects of (1) the cost of dispersal (2) the deme size and (3) the shape of the habitat under a limited number of constraints. The same methods, however, could be used to study the evolution of dispersal distance under more specific constraints. For example, one may derive the ES allocation among two types of offspring that have different dispersal abilities (e.g. Harada, 1999), as may result from seed heteromorphism (e.g. Venable, 1985). Specific modes of dispersal may impose constraints on the form of dispersal distributions, and the possible distributions under such 
constraints may be characterized by some parameters (e.g. Neubert et al., 1995; Tufto et al., 1997). Then, the optimal strategy will be found by considering a fitness measure that is a weighted average of the selection pressures at each distance, where the weights are determined by the effects of each parameter on dispersal at a given distance. Such analyses would be of interest given data on cost of dispersal, and a known mechanism of dispersal. Although one can identify constraints on the shape of a dispersal distribution, imposed by specific dispersal mechanisms, the mechanisms of dispersal in natural populations of a given species are probably a mixture of several of the processes considered in mechanistic models of dispersal. We would need more knowledge of actual constraints in such cases. Renewed interest for analyses of dispersal distributions (e.g. Clark et al., 1999, Stoyan \& Wagner 2001) may provide some advances in this direction.

The above analysis, however, relies on the assumption that the habitat is homogeneous. Currently, no exact condition for convergence stability (comparable with eq. 3 ) is available for spatially and temporally heterogeneous populations evolving under localized dispersal. Some insights may be obtained from analytical expressions derived from pair-approximations but these expressions cannot be interpreted with the same confidence as exact expressions without thorough checking by simulation (see, e.g. multiple reviews in Dieckmann et al., 2000). Therefore, individual based simulations are required to provide a better understanding of the multiplicity of factors acting on the evolution of dispersal distributions. Our model could serve as a reference against which the importance of the additional factors included in these simulations can be evaluated.

\section{Acknowledgments}

We thank É. Imbert, I. Olivieri, O. Ronce, T. Kawecki and S. West for comments. SG gratefully acknowledges support from The Wellcome Trust (grant 62429) and the British Council. This is paper ISEM 02-027.

\section{Supplementary material}

The following material is available from http://www. blackwell-science.com/products/journals/suppmat/JEB/JE B430/JEB430sm.htm

\section{Supplementary Appendix}

\section{References}

Bengtsson, B.O. 1978. Avoiding inbreeding: at what cost? J. Theor. Biol. 73: 439-444.

Bolker, B.M. \& Pacala, S.W. 1999. Spatial moment equations for plant competition: understanding spatial strategies and the advantages of short dispersal. Am. Nat. 153: 575602 .
Clark, J.S., Lewis, M. \& Horvath, L. 2001. Invasion by extremes: population spread with variation in dispersal and reproduction. Am. Nat. 157: 537-554.

Clark, J.S., Silman, M., Kern, R., Macklin, E. \& HilleRisLambers, J. 1999. Seed dispersal near and far: patterns accross temperate and tropical forests. Ecology 80: 1475-1494.

Comins, H.N. 1982. Evolutionarily stable strategies for localized dispersal in two dimensions. J. Theor. Biol. 94: 579-606.

Dieckmann, U., Law, R. \& Metz, J.A.J., eds. 2000. The Geometry of Ecological Interactions: Simplifying Spatial Complexity. Cambridge University Press, Cambridge.

Endler, J.A. 1977. Geographical Variation, Speciation, and Clines. Princeton University Press, Princeton, NJ, USA.

Ezoe, H. 1998. Optimal dispersal range and seed size in a stable environment. J. Theor. Biol. 190: 287-293.

Gandon, S., Capowiez, Y., Dubois, Y., Michalakis, Y. \& Olivieri, I. 1996. Local adaptation and gene-for-gene coevolution in a metapopulation model. Proc. Roy. Soc. (Lond.) B 263: 1003-1009.

Gandon, S. \& Rousset, F. 1999. Evolution of stepping stone dispersal rates. Proc. Roy. Soc. (Lond.) B 266: 2507-2513.

Hamilton, W.D. 1964. The genetical evolution of social behavior. I. J. Theor. Biol. 7: 1-16.

Hamilton, W.D. \& May, R. 1977. Dispersal in stable habitats. Nature 269: 578-581.

Harada, Y. 1999. Short- vs. long-range disperser: the evolutionarily stable allocation in a lattice-structured habitat. J. Theor. Biol. 201: 171-187.

Hastings, W.K. 1970. Monte Carlo sampling methods using Markov chains and their applications. Biometrika 57: 97-109.

Hovestadt, T., Messner, S. \& Poethke, H.J. 2001. Evolution of reduced dispersal mortality and 'fat-tailed' dispersal kernels in autocorrelated landscapes. Proc. Roy. Soc. (Lond.) B 268: 385-391.

Janzen, D.H. 1970. Herbivores and the number of tree species in tropical forests. Am. Nat. 104: 501-528.

Kaltz, O. \& Shykoff, J.A. 1998. Local adaptation in host-parasite systems. Heredity 81: 361-370.

Kot, M., Lewis, M.A. \& van den Driessche, P. 1996. Dispersal data and the spread of invading organisms. Ecology 77: 20272042.

Mollison, D. 1991. Dependence of epidemic and population velocities on basic parameters. Math. Biosciences 107: 255-287.

Neubert, M.G., Kot, M. \& Lewis, M.A. 1995. Dispersal and pattern formation in a discrete-time predator-prey model. Theor. Popul. Biol. 48: 7-43.

Olivieri, I. \& Berger, A. 1985. Seed dimorphism for dispersal: physiological, genetic and demographical aspects. In: Genetic Differentiation and Dispersal in Plants (P. Jacquard, G. Heim \& J. Antonovics, eds), pp. 413-429. Springer-Verlag, Berlin.

Packer, A. \& Clay, K. 2000. Soil pathogens and spatial patterns of seedling mortality in a temperate tree. Nature 404: 278-281.

Portnoy, S. \& Willson, M.F. 1993. Seed dispersal curves: behavior of the tails of the distribution. Evol. Ecol. 7: 25-44.

Ronce, O., Olivieri, I., Clobert, J. \& Danchin, É. 2001. Perspectives on the study of dispersal evolution. In: Dispersal (J. Clobert, É. Danchin, A. A. Dhondt \& J. D. Nichols, eds), pp. 341-357. Oxford University Press, Oxford.

Ronsheim, M.L. 1997. Distance-dependent performance of asexual progeny in Allium vineale (Liliaceae). Am. J. Bot. 84: 1279-1284.

Rousset, F. 1997. Genetic differentiation and estimation of gene flow from $F$-statistics under isolation by distance. Genetics 145 : 1219-1228. 
Rousset, F. \& Billiard, S. 2000. A theoretical basis for measures of kin selection in subdivided populations: finite populations and localized dispersal. J. Evol. Biol. 13: 814-825.

Savill, N.J. \& Hogeweg, P. 1998. Spatially induced speciation prevents extinction: the evolution of dispersal distance in oscillatory predator-prey models. Proc. Roy. Soc. (Lond.) B 265: $25-32$.

Shigesada, N. \& Kawasaki, K. 1997. Biological Invasions: Theory and Practice. Oxford University Press, Oxford.

Slatkin, M. 1987. Gene flow and the geographic structure of natural populations. Science 236: 787-792.

Stamp, N.E. \& Lucas, J.R. 1983. Ecological correlates of explosive seed dispersal. Oecologia 59: 272-278.

Stoyan, D. \& Wagner, S. 2001. Estimating the fruit dispersion of anemochorous forest trees. Ecol. Modelling 145: 35-47.

Taylor, P.D. 1988. An inclusive fitness model for dispersal of offspring. J. Theor. Biol. 130: 363-378.

Tufto, J., Engen, S. \& Hindar, K. 1997. Stochastic dispersal processes in plant populations. Theor. Popul. Biol. 52: 16-26.

Van Valen, L. 1971. Group selection and the evolution of dispersal. Evolution 25: 591-598.

Venable, D.L. 1985. The evolutionary ecology of seed heteromorphism. Am. Nat. 126: 577-595.

Venable, D.L. \& Brown, J.S. 1988. The selective interactions of dispersal, dormancy, and seed size as adaptations for reducing risk in variable environments. Am. Nat. 131: 360-384.

Waser, N.M. 1993. Population structure, optimal outbreeding, and assortative mating in angiosperms. In: The Natural History of Inbreeding and Outbreeding (N. W. Thornhill, ed.), pp. 173199. University Chicago Press, Chicago.

Willson, M.F. 1993. Dispersal mode, seed shadows, and colonization patterns. Vegetatio 107: 261-280.

Wolfram, S. 1999. The Mathematica Book, 4th edn. Wolfram Media/Cambridge University Press, Cambridge.

Received 1 March 2002; accepted 12 April 2002

\section{Appendix}

\section{Selection on different kinds of mutants}

The coevolution of dispersal probabilities at all distances is evaluated by first considering mutants which affect one dispersal probability $d_{x, y}$ and affect proportionally all other dispersal probabilities. By definition, such mutants do not affect the relative dispersal probabilities $f_{\mathbf{j}}$ among genes that do not disperse at distance $D$. As noted in the main text, the mutant and resident strategies can each be described by a single variable $z$. This variable is the dispersal probability at distance $D$. The dispersal probability at any other distance $\mathbf{j}$ is $(1-z) f_{\mathbf{j}}$. In this case the relative numbers of juveniles from deme $\mathbf{i}$ in competition for deme $\mathbf{j}$ are

$g_{\mathbf{i} \rightarrow \mathbf{j}}\left(z_{\mathbf{i}}, D\right)= \begin{cases}\left(1-c_{\mathbf{i}-\mathbf{j}}\right) d_{\mathbf{i}-\mathbf{j}}(z)=\left(1-c_{D}\right) z_{D} & \text { if } \mathbf{i}-\mathbf{j}=D \\ \left(1-c_{\mathbf{i}-\mathbf{j}}\right) f_{\mathbf{i}-\mathbf{j}}\left(1-z_{D}\right) & \text { otherwise }\end{cases}$

Selection on more general forms of mutational effects on the dispersal distribution can then be measured as follows. For each distance $D$, the restricted form of mutants considered above defines one vector of mutational effects $\mathbf{e}_{D}$. The vectors $\left(\mathbf{e}_{\mathbf{0}}, \ldots\right)$ form a basis in which any more general form of mutant can be described by a linear combination $\sum_{D} q_{D} \mathbf{e}_{D}$. Since differentiation is a linear operation, the fitness measure $\phi$ for any mutant can then be written as $\sum_{D} q_{D} \phi_{D}\left(z^{a}\right)$. The strategy $\mathbf{d}^{a}$ is stable against any mutant only if $\phi \leq 0$ for any mutant. Thus, the stability of $\mathbf{d}^{a}$ can be deduced from the values of $\phi_{D}\left(z^{a}\right)$ for the more restricted form of mutants. In particular, for any $D, D^{\prime}$ such that $d_{D}^{a}>0$ and $d_{D^{\prime}}^{a}>0$, it requires that $\phi_{D}=\phi_{D^{\prime}}=0$.

\section{Finding the ES dispersal distribution}

\section{Fourier analysis}

We use Fourier analysis as in earlier work; see Rousset $\delta$ Billiard (2000) and the electronic Appendix of Gandon $\delta$ Rousset (1999) for general background and related results. Consider two vectors $\mathbf{q} \equiv\left(q_{x}, q_{y}\right)$ and $\boldsymbol{\rho} \equiv\left(\rho_{x}, \rho_{y}\right)$. For any suitable function $h$ let

$$
\mathscr{F}_{\boldsymbol{\rho}}(h) \equiv \sum_{\mathbf{q}} h(\mathbf{q}) e^{\mathbf{i q} \cdot \boldsymbol{\rho}}
$$

be its Fourier transform in $\boldsymbol{\rho}$, where $1=\sqrt{-1}$ and $\mathbf{q} \cdot \boldsymbol{\rho}$ is simply the scalar product of the two vectors, so that $e^{i \mathbf{q} \cdot \boldsymbol{\rho}}=e^{1 q_{x} \boldsymbol{\rho}_{x}} e^{1 q_{y} \boldsymbol{\rho}_{y}}$. Sums over $\mathbf{q}$ are sums over all possible vectorial distances on the lattice, $q_{x}=0, \ldots, n_{x}-1$, $q_{y}=0, \ldots, n_{y}-1$. For any vector $\mathbf{q}$, let $\boldsymbol{\theta} \equiv \boldsymbol{\theta}(\mathbf{q}) \equiv$ $2 \pi\left(q_{x} / n_{x}, q_{y} / n_{y}\right)$. For any suitable function $h$ let

$$
\mathscr{L}_{\mathbf{j}}(h) \equiv \frac{1}{n} \sum_{\mathbf{q}} h(\boldsymbol{\theta}) e^{-1 \boldsymbol{\theta} \cdot \mathbf{j}}
$$

be its inverse Fourier transform in j. Let $\psi(\boldsymbol{\rho}) \equiv$ $\sum_{\mathbf{q}} m_{\mathbf{q}} e^{\mathbf{i q} \cdot \boldsymbol{\rho}}$ be the characteristic function of the distribution of backward dispersal distance.

\section{An algorithm giving the ESS}

Here we give a relationship between the Fourier transform of the dispersal distribution and some functions of the costs at different distances. To get an expression only in terms of the backward dispersal probabilities and of their transform, we first consider the average cost paid by dispersing juveniles,

$$
\bar{c} \equiv \sum_{\mathbf{i}} c_{\mathbf{i}} d_{\mathbf{i}}
$$

It is shown in the Supplementary Appendix that an ES dispersal distribution $\psi$ will be a solution of the recursion giving the next iteration distribution $\psi^{\prime}$ as

$$
\psi^{\prime}(\boldsymbol{\rho})=\frac{\left(1-\frac{1}{N n}\right) \sum_{\mathbf{q}} \frac{1-\bar{c}}{1-c_{\mathbf{q}}} m_{\mathbf{q}} e^{i \mathbf{q} \cdot \boldsymbol{\rho}}+\frac{1}{N n} \sum_{\mathbf{q} \neq \mathbf{0}} \frac{\psi(\boldsymbol{\theta}) \psi(\boldsymbol{\rho}-\boldsymbol{\theta})}{1-\psi^{2}(\boldsymbol{\theta})}}{1-\frac{1}{N n}+\frac{1}{N n} \sum_{\mathbf{q} \neq \mathbf{0}} \frac{\psi^{2}(\boldsymbol{\theta})}{1-\psi^{2}(\boldsymbol{\theta})}}
$$

Thus, one may seek the ESS by iterating this recursion. Note that $\bar{c}$ and the $m$ s must be recomputed at each 
iteration. Convergence was checked by examination of the $\phi$ s. However, convergence of the recursion to the solution is not guaranteed (it is not logically related to evolutionary convergence stability). In practice, the recursion is often found to diverge, but recursions that converge are deduced from the above one: we considered recursions of the form $\psi^{\prime}(\boldsymbol{\rho})=(1-\epsilon)$ $\psi(\boldsymbol{\rho})+\epsilon \times($ right hand part of the above equation), for some suitable value of $\epsilon$.

This algorithm has the interesting property that if dispersal is zero at some distance $D$ at iteration $t$, it will be zero at this distance at iteration $t+1$. Thus one may constrain the set of possible dispersal distributions by imposing an initial distribution with strictly nonzero dispersal within and only within this set. The algorithm will then converge to the optimal distribution given the constraint of zero dispersal outside this set, i.e. the distribution with $\phi_{D}=0$, or zero dispersal and $\phi_{D}<0$, within this set. On the other hand, it is necessary to initiate the algorithm with nonzero dispersal everywhere (e.g. an island mode of dispersal) in order to be sure to obtain the unconstrained optimum. 